\section{A Rare Case of Multifocal Giant Gell Tumor of the Tendon Sheath Involving Both the Hand and the Foot}

Seong Jae Hong ${ }^{1}$, Jong Ick Whang ${ }^{2}$, Hyeung Gyo Seo ${ }^{2}$, Su Rak Eo ${ }^{1}$, Sang Hun Cho ${ }^{1}$

${ }^{1}$ Department of Plastic and Reconstructive Surgery, Dongguk University Ilsan Hospital, Dongguk University Graduate School of Medicine, Goyang; ${ }^{2}$ Department of Plastic and Reconstructive Surgery, Duson Hospital, Ansan, Korea

\author{
Correspondence: Jong Ick Whang \\ Department of Plastic and Reconstructive Surgery, Duson Hospitat, \\ 114 Seonbugwangjang 1-ro, Danwon-gu, Ansan 425-141, Korea \\ Tel: +82-31-402-0114-207, Fax: +82-31-402-1805 \\ E-mail: hand4u@chol.com \\ No potential conflict of interest relevant to this article was reported. \\ Received: 29 Aug 2013 • Revised: 12 Sep 2013 • Accepted: 13 Sep 2013 \\ pISSN: 2234-6163 • elSSN: 2234-6171 \\ http://dx.doi.org/10.5999/aps.2013.40.6.795 • Arch Plast Surg 2013;40:795-797 \\ Copyright (C) 2013 The Korean Society of Plastic and Reconstructive Surgeons \\ This is an Open Access article distributed under the terms of the Creative Commons \\ Attribution Non-Commercial License (http://creativecommons.org/licenses/by-nc/3.0/) \\ which permits unrestricted non-commercial use, distribution, and reproduction in any \\ medium, provided the original work is properly cited.
}

The giant cell tumor of the tendon sheath (GCTTS) is a relatively common entity, and it is the second most frequent soft tissue tumor affecting the hand followed by ganglion cyst. It is clinically characterized by an asymptomatic, solitary, slowly-growing, palpable subcutaneous benign mass localized to the palmar aspect of the extremities adjacent to the distal interphalangeal (IP) joint. Its onset takes several months to years $[1,2]$

According to a review of literature, there are rare cases of multifocal origin of the tumor. More recent studies have reported a small number of cases of multifocal GCTTS [3].

We experienced a rare case of multifocal GCTTS with different types occurring simultaneously at two different sites (the upper and lower extremities) in the same patient.

A 59-year-old woman presented with a slowlygrowing mass of the right thumb and left plantar region, and the patient concurrently had an increasingly symptomatic, soft tissue swelling. The patient had neither sensory disturbances nor stiffness of the IP joint of the thumb. The interval between onset of symptoms and first visit to our clinic was three years.

On physical examination, the patient had a mass on the plantar surface of the left foot as well as the radial

aspect of the IP joint and the proximal phalanx of the right thumb. Both masses were solid, well-defined, and slightly tender. Mass was measured to be $2 \mathrm{~cm}$ on right thumb, $1.5 \mathrm{~cm}$ on left plantar region, respectively. On plain radiography of the right thumb, the mass showed a well-defined soft-tissue shadow. There were neither periosteal reactions nor articular involvement (Fig. 1). On plain radiography of the lesion on the left plantar region, there were no notable findings.

Surgery was performed under general anesthesia with tourniquet control, using magnifying loupe.

Bruner's incision to the volar aspect skin of the thumb was performed at the level of proximal phalanx. A meticulous dissection was performed with caution to achieve a complete resection of the tumor. Thus, the diaphysis of the right proximal phalanx was exposed. The mass had contact to neurovascular structures as well as both extensor pollicis longus (EPL) and flexor pollicis longus (FPL) tendons. The mass was removed along with the capsule. It was unavoidable, however,

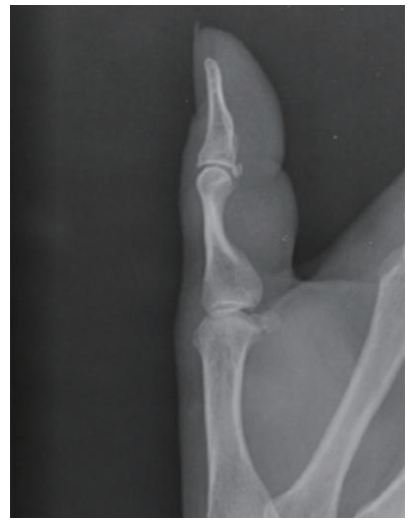

Fig. 1.

A lateral view of plain radiograph of the primary lesion on the right proximal phalanx level of the thumb shows a well-defined soft tissue contour. There were neither periosteal reactions nor articular involvement.

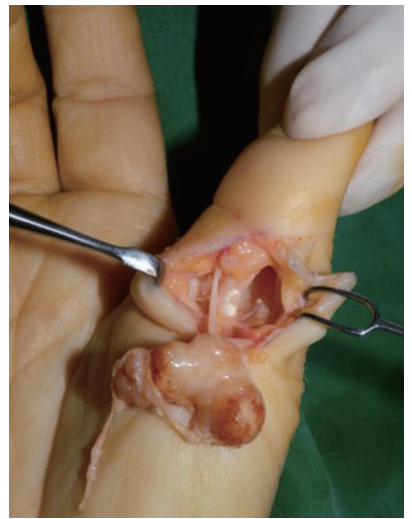

Fig. 2.

Grossly, the lesion is a multilobulated, kidney shaped mass that is surrounded by pseudocapsule and yellowish in color, and it is measured as $2 \times 1.5 \times 1.5 \mathrm{~cm}$.

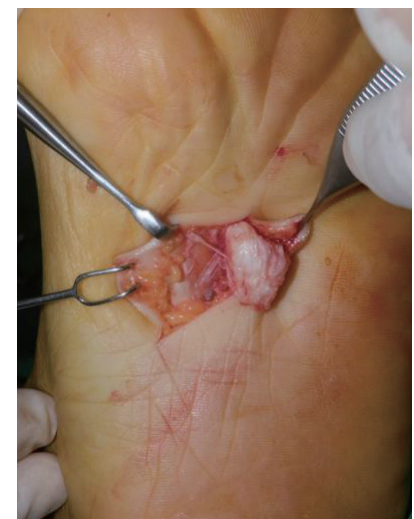

Fig. 3.

The lesion of the foot, releasing the plantar fascia after mass extirpation, has no bone pressure effect on metatarsal bones. 

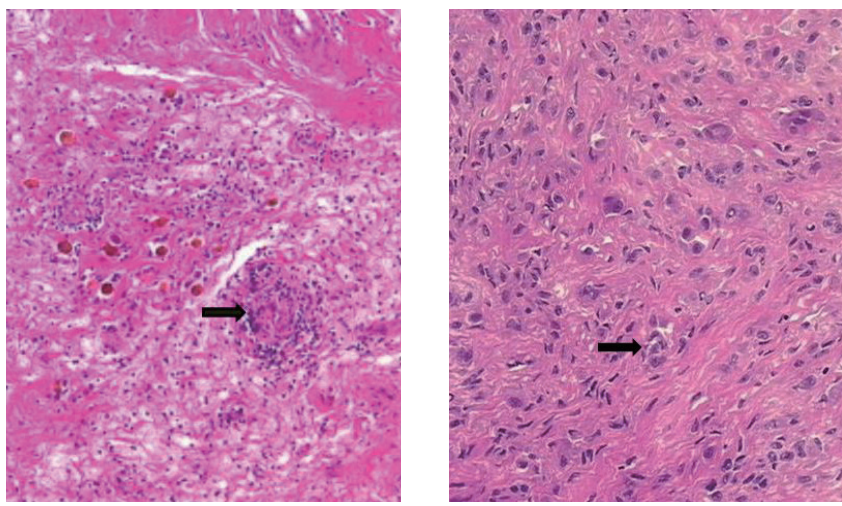

Fig. 4.

On histopathology, the mass involving the hand is composed of multinucleated giant cells (black arrow), histiocytes and hemosiderin deposits which

is surrounded by fibrous capsule. These findings establish a diagnosis of giant cell tumor $\left(H \& E_{1} \times 200\right)$.
Fig. 5.

On histopathology, the mass involving the foot is composed of less multinucleated giant cells (black arrow) but more hypercellular $(\mathrm{H} \& \mathrm{E}, \times 400)$. to perform a partial excision of the tendon sheath of both EPL and FPL for complete removal. Grossly, the lesion was a nodular type, multi-lobulated, and kidney shaped mass that is surrounded by pseudocapsule and yellowish in color, and it was measured as $2 \times 1.5 \times 1.5$ $\mathrm{cm}$ (Fig. 2). The diffuse type mass involving the plantar fascia of the foot, had no bone involvement, and it was measured as $1.5 \times 1.5 \times 1.5 \mathrm{~cm}$ (Fig. 3). Both lesions were primarily closed without tension.

On histopathology, both masses involving both the hand and the foot are composed of multinucleated giant cells, histiocytes and hemosiderin deposits. These findings established a diagnosis of giant cell tumor (Figs. 4, 5).

There was no metastatic disease on chest radiography or chest magnetic resonance imaging. The patient had a good range of active movement without further symptoms, stiffness and a substantial loss of function. The patient was followed up with a plain radiography after postoperative 3 and 6 months without recurrence.

The GCTTS is a non-neoplastic benign lesion of giant cells occurring in the adjacent areas to the joint. On plain radiography, it is identified as soft-tissue contour that potentially causes a pressure-induced erosion of the cortex in the adjacent area [1].

There are rare cases of multifocal lesions of GCTTS. According to a retrospective study that was conducted in a consecutive series of 207 patients, there was only one patient who developed lesions at two different sites: the right ring finger and the left great toe [2]. Altaykan et al. [3] reported a case of GCTTS occur- ring simultaneously at two different sites on the flexor digitorum superficialis tendon of the little finger [3]. There is still a controversy as to the etiology and the pathogenesis of GCTTS. Therefore, its multifocal presence also remains unclear. Clinically based on histopathology, differential diagnoses of GCTTS include ganglion cyst, myxoid cyst, dermatofibroma, lipoma, glomus tumor, hemangioma, neurofibroma, foreign bodies, sweat gland tumor, synovial carcinoma, giant cell tumor of the soft tissue and bone, fibroma and rheumatoid nodules and primitive neuroectodermal tumor $[1,2]$.

GCTTS is commonly characterized by adhesion to the extensor or flexor tendon sheath. It is such a relatively small-sized mass that its diameter rarely exceeds $5 \mathrm{~cm}$. The most possible pathogenesis of GCTTS is an inflammatory response accompanied by reactive or regenerative hyperplasia due to chronic antigen exposure or a trauma history [4].

The treatment goals are to eliminate the lesions through a meticulous resection and to preserve the functions of the affected tendon and adjacent structures. With the development of operating microscopes and magnifying loupes, it has become easier not only to achieve a radical excision but also to prevent its recurrence [5]. A complete excision of the lesions might pose challenging problems for surgeons because they are located close to, or often involve, the tendon and neurovascular structures. Surgeons should avoid accidentally puncturing the lesion because potential seeding to adjacent soft-tissue structures may lead to the recurrence. It has been reported that the local recurrence after surgical excision occurred in up to $44 \%$ of total cases. Overall recurrence rate in patients with GCTTS reaches up to $27 \%$ at 2 years and 3 months on average after resection. Its recurrence had no significant correlation with GCTTS in a specific finger or phalanx [4].

We experienced a rare, multifocal case of GCTTS, nodular type involving both the EPL and FPL tendon, at the right proximal phalanx level and another diffuse type involving the left plantar fascia.

In conclusion, our case indicates that it would be mandatory to evaluate the possibility of multifocal GCTTS in patients with soft-tissue swelling of the hand and the difference of tumor recurrence by type of giant cell tumor should bourn in mind.

\section{References}

1. Stoller DW, Tirman PF, Bredella MA. Diagnostic 
imaging: orthopaedics. Salt Lake: Amirsys; 2004.

2. Ushijima M, Hashimoto H, Tsuneyoshi M, et al. Giant cell tumor of the tendon sheath (nodular tenosynovitis). A study of 207 cases to compare the large joint group with the common digit group. Cancer 1986;57:875-84.

3. Altaykan A, Yildiz K, Hapa O, et al. Multifocal giant cell tumor of the tendon sheath occuring at different localizations of the same tendon of a finger: a case report and review of the literature. Eklem Hastalik Cerrahisi 2009;20:119-23.

4. Reilly KE, Stern PJ, Dale JA. Recurrent giant cell tumors of the tendon sheath. J Hand Surg Am 1999;24:1298-302.

5. Ikeda K, Osamura N, Tomita K. Giant cell tumour in the tendon sheath of the hand: importance of the type of lesion. Scand J Plast Reconstr Surg Hand Surg 2007;41:138-42.

\section{Correction of Severe} "Pixie Ear" Deformity after Rhytidectomy with Modified Minimal Access Cranial Suspension Lift

Stamatis Sapountzis ${ }^{1}$, Kidakorn Kiranantawat ${ }^{2}$, Pericles Foroglou ${ }^{3}$, Achilleas Chantes ${ }^{4}$, Antonios Antoniou ${ }^{3}$, Dimitrios Dionyssiou ${ }^{3}$, Leonidas Pavlidis ${ }^{3}$, Efterpi Demiri ${ }^{3}$

${ }^{I}$ Department of Plastic and Reconstructive Surgery, China Medical University Hospital, Taichung, Taiwan; ${ }^{2}$ Division of Plastic and Maxillofacial Surgery, Ramathibodi Hospital, Mahidol University, Bangkok, Thailand; ${ }^{3}$ Department of Plastic Surgery, Aristotle University Thessaloniki, Thessaloniki; ${ }^{4}$ Department of Plastic Surgeon, Private Paractice, Thessaloniki, Greece

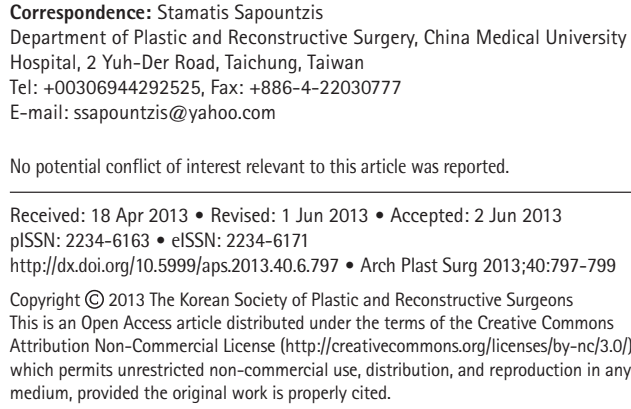

The facelift remains the gold standard in facial rejuvenation, with a high satisfaction rate. However, excessive skin excision of the skin flap can produce unsatisfactory results and minor or even major complications.
Amongst these complications, the pixie ear deformity can be recognized by its "stuck on" or "pulled" appearance, which is caused by the extrinsic pull of the medial cheek skin and jaw-line flaps at the earlobe attachment point, the otobasion inferius. Following rhytidectomy, the tension results in migration of the earlobe attachment point (otobasion inferius) from a posterior cephalad position to an anterior caudal position [1]. It is also possible that the pixie ear deformity can be accompanied by other tension-related complications such as a sweeping effect, scar migration, unnatural appearance of the tragus, and a "face-lift look."

In the medical literature, several techniques have been described for correction of this clinical condition. However, disadvantages such as extra scars on the neck or the earlobe and the controversial effectiveness of minimally invasive techniques in severe pixie ear deformities make the correction of this complication challenging to achieve. In this report, we present a case of severe pixie ear deformity after rhytidectomy, which was alleviated with a modification of the minimal access cranial suspension (MACS) lift.

A 53-year-old woman presented to our clinic totally dissatisfied with the postoperative result of a previous typical rhytidectomy performed elsewhere one year previously. Her main complaint was the unnatural appearance of the periauricular area. On clinical examination, severe pixie ear deformity and anterior migration of the preauricular scar were noted (Fig. 1). These facelift stigmata were the consequences of a rhytidectomy with overesection of the skin flaps, resulting in distortion of the position of the earlobe, which was attached to the angle of the mandible. With
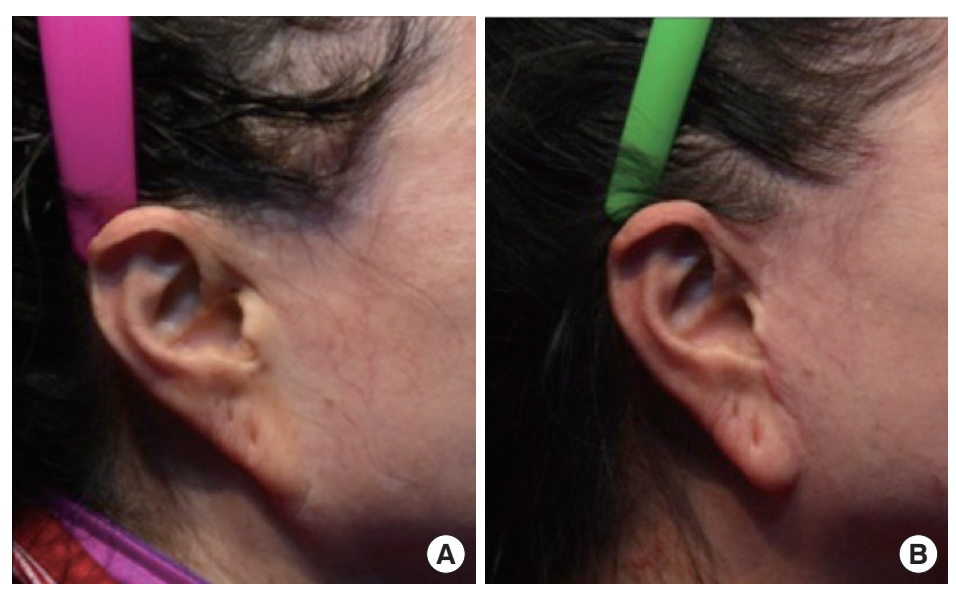

Fig. 1.

(A) Severe pixie ear deformity after a facelift. (B) Postoperative result at 18 months of follow-up. 